\title{
A NOTE ON A RESULT OF RUZSA, II
}

\author{
MIN TANG
}

(Received 21 February 2010)

\begin{abstract}
Let $\sigma_{A}(n)=\left|\left\{\left(a, a^{\prime}\right) \in A^{2}: a+a^{\prime}=n\right\}\right|$, where $n \in \mathbb{N}$ and $A$ is a subset of $\mathbb{N}$. Erdôs and Turán conjectured that for any basis $A$ of $\mathbb{N}, \sigma_{A}(n)$ is unbounded. In 1990, Ruzsa constructed a basis $A \subset \mathbb{N}$ for which $\sigma_{A}(n)$ is bounded in square mean. Based on Ruzsa's method, we proved that there exists a basis $A$ of $\mathbb{N}$ satisfying $\sum_{n \leq N} \sigma_{A}^{2}(n) \leq 1449757928 N$ for large enough $N$. In this paper, we give a quantitative result for the existence of $N$, that is, we show that there exists a basis $A$ of $\mathbb{N}$ satisfying $\sum_{n \leq N} \sigma_{A}^{2}(n) \leq 1069693154 N$ for $N \geq 7.628517798 \times 10^{27}$, which improves earlier results of the author ['A note on a result of Ruzsa', Bull. Aust. Math. Soc. 77 (2008), 91-98].
\end{abstract}

2000 Mathematics subject classification: primary 11B13.

Keywords and phrases: Erdős-Turán conjecture, basis.

\section{Introduction}

For $A, B \subseteq \mathbb{Z}$ and $n \in \mathbb{Z}$, let

$$
\begin{aligned}
\sigma_{A, B}(n) & =|\{(a, b) \in A \times B: a+b=n\}|, \\
\delta_{A, B}(n) & =|\{(a, b) \in A \times B: a-b=n\}| .
\end{aligned}
$$

Let $\sigma_{A}(n)=\sigma_{A, A}(n)$ and $\delta_{A}(n)=\delta_{A, A}(n)$. A subset $A$ of $\mathbb{N}$ is called a basis of $\mathbb{N}$ if $\sigma_{A}(n) \geq 1$ for $n \geq n_{0}$. In 1941, Erdős and Turán [3] formulated the following attractive conjecture.

Conjecture (Erdős-Turán). If $A \subset \mathbb{N}$ is a basis of $\mathbb{N}$, then $\sigma_{A}(n)$ cannot be bounded:

$$
\limsup _{n \rightarrow+\infty} \sigma_{A}(n)=+\infty
$$

This harmless-looking conjecture seems to be extremely difficult. In 1954, using probabilistic methods, Erdős [2] proved the existence of a basis of $\mathbb{N}$ for which $\sigma(n)$ satisfies $c_{1} \log n<\sigma(n)<c_{2} \log n$ for all $n$ with certain positive constants $c_{1}, c_{2}$. In 1990, Ruzsa [5] constructed a basis $A$ of $\mathbb{N}$ for which $\sigma_{A}(n)$ is bounded in mean

The author was supported by the National Natural Science Foundation of China, Grant No. 10901002.

(C) 2010 Australian Mathematical Publishing Association Inc. 0004-9727/2010 \$16.00 
square, that is, he constructed a basis $A$ satisfying $\sum_{n \leq N} \sigma_{A}^{2}(n)=O(N)$. Based on Ruzsa's method, Tang [6] proved that there exists a basis $A$ of $\mathbb{N}$ satisfying $\sum_{n \leq N} \sigma_{A}^{2}(n) \leq 1449757928 N$ for large enough $N$.

In this paper, improving Ruzsa's method and employing a result concerning the function $\pi(x)$ of Panaitopol, we give a quantitative result for the existence of $N$ and obtain a stronger version of the above result.

THEOREM 1.1. There exists a set A of nonnegative integers that forms a basis of $\mathbb{N}$, and satisfies $\sum_{n \leq N} \sigma_{A}^{2}(n) \leq 1069693154 N$ for $N \geq 7.628517798 \times 10^{27}$.

Throughout this paper, let $p$ be an odd prime, $\mathbb{Z}_{p}$ be the set of residue classes $\bmod p$ and $G=\mathbb{Z}_{p}^{2}$. For $A, B \subseteq G$, let $A-B=\{a-b: a \in A, b \in B\}$. Denote $Q_{k}=\left\{\left(u, k u^{2}\right): u \in \mathbb{Z}_{p}\right\} \subset G$ and for a finite set $A$, let

$$
D(A)=\sum_{-\infty}^{+\infty} \sigma_{A}^{2}(n)=\left|\left\{(a, b, c, d) \in A^{4}: a+b=c+d\right\}\right|
$$

$\varphi$ is a mapping

$$
\varphi: G \rightarrow \mathbb{Z}, \quad \varphi(a, b)=a+2 p b,
$$

where we identify the residues mod $p$ with the integers $0 \leq j \leq p-1$.

\section{Proofs}

LEMMA 2.1. For any real number $x \geq 1342$, there exists at least one prime in the interval $(x, 1.0147 x]$.

PROOF. By direct calculation we know that Lemma 2.1 is true for $1342 \leq x \leq$ 1341755571000 .

We now assume that $x>1341755571000$. We employ a result concerning the function $\pi(x)$ of Panaitopol [4]. That is,

$$
\pi(x)<\frac{x}{\log x-1-(\log x)^{-0.5}} \quad \forall x \geq 6 .
$$

and

$$
\pi(x)>\frac{x}{\log x-1+(\log x)^{-0.5}} \quad \forall x \geq 59 .
$$

Thus it suffices to prove that for $x>1341755571000$,

$$
\begin{aligned}
& \pi(1.0147 x)-\pi(x) \\
& \quad>\frac{1.0147 x}{\log (1.0147 x)-1+(\log (1.0147 x))^{-0.5}}-\frac{x}{\log x-1-(\log x)^{-0.5}} \\
& \quad \geq 0 .
\end{aligned}
$$

This is equivalent to showing that

$147 \log x \geq 147+10^{4} \log 1.0147+10147(\log x)^{-0.5}+10^{4}(\log x+\log 1.0147)^{-0.5}$. 
It is easy to verify that the inequality is true for $x=1341755571000$. Hence the inequality is true for $x>1341755571000$.

This completes the proof of Lemma 2.1 .

Lemma 2.2 [6, Lemma 2]. For $g=(a, b) \in G$, and fixed $k, l \in \mathbb{Z}_{p} \backslash\{0\}$, consider the equation

$$
g=x-y, \quad x \in Q_{k}, y \in Q_{l} .
$$

If $k-l \neq 0$, this equation is solvable unless

$$
\left(\frac{(k-l) b+k l a^{2}}{p}\right)=-1,
$$

and it has at most two solutions. If $k-l=0$, it has at most one solution except for $g=0$, when it has $p$ solutions.

LEMMA 2.3. Let $p(\geq 11)$ be prime and $m$ be a quadratic nonresidue of $p$ with $m+1 \not \equiv 0 \bmod p, \quad 3 m+1 \not \equiv 0 \bmod p$ and $m+3 \not \equiv 0 \bmod p . \quad$ Put $B=Q_{m+1} \cup$ $Q_{m(m+1)} \cup Q_{2 m}$. Then $1 \leq \sigma_{B}(g) \leq 16$ for all $g \in G$ and $1 \leq \delta_{B}(g) \leq 11$ for all $g \neq 0$.

Proof. The statement that $1 \leq \sigma_{B}(g) \leq 16$ for all $g \in G$ is obtained by Yong-Gao Chen in [1, Lemma 2]. We now show that $1 \leq \delta_{B}(g) \leq 11$ for all $g \neq 0$.

Suppose that there is a $g=(a, b) \in G, g \notin Q_{2 m}-Q_{m+1}, g \notin Q_{m(m+1)}-Q_{2 m}$. Note that $m$ is a quadratic nonresidue of $p$, hence $m-1 \not \equiv 0 \bmod p$ and, by Lemma 2.2,

$$
\left(\frac{(m-1) b+2 m(m+1) a^{2}}{p}\right)=-1, \quad\left(\frac{m(m-1) b+2 m^{2}(m+1) a^{2}}{p}\right)=-1 .
$$

Thus

$$
1=\left(\frac{(m-1) b+2 m(m+1) a^{2}}{p}\right)^{2}\left(\frac{m}{p}\right)=\left(\frac{m}{p}\right)=-1 .
$$

This contradiction shows that

$$
G=\left(Q_{2 m}-Q_{m+1}\right) \cup\left(Q_{m(m+1)}-Q_{2 m}\right),
$$

which is stronger than the required $B-B=G$.

Let

$$
T=\{m+1, m(m+1), 2 m\} .
$$

If $g=(a, b) \in G(g \neq 0)$, then $(m-1) b$ cannot equal both $2 m(m+1) a^{2}$ and $-2 m(m+1) a^{2}$. Now we consider the following three cases.

Case 1. $(m-1) b \neq 2 m(m+1) a^{2}$ and $(m-1) b \neq-2 m(m+1) a^{2}$. Then we have $g \notin\left(Q_{m+1}-Q_{2 m}\right) \cap\left(Q_{2 m}-Q_{m(m+1)}\right)$ and $g \notin\left(Q_{2 m}-Q_{m+1}\right) \cap\left(Q_{m(m+1)}-Q_{2 m}\right)$. 
Indeed, if $g \in\left(Q_{m+1}-Q_{2 m}\right) \cap\left(Q_{2 m}-Q_{m(m+1)}\right)$, by $(m-1) b \neq 2 m(m+1) a^{2}$ we have

$$
\left(\frac{(-m+1) b+2 m(m+1) a^{2}}{p}\right)=1, \quad\left(\frac{\left(-m^{2}+m\right) b+2 m^{2}(m+1) a^{2}}{p}\right)=1 .
$$

Thus

$$
1=\left(\frac{(-m+1) b+2 m(m+1) a^{2}}{p}\right)^{2}\left(\frac{m}{p}\right)=\left(\frac{m}{p}\right)=-1 .
$$

Similarly, by $(m-1) b \neq-2 m(m+1) a^{2}$, we can show that

$$
g \notin\left(Q_{2 m}-Q_{m+1}\right) \cap\left(Q_{m(m+1)}-Q_{2 m}\right) .
$$

Hence, for $g \neq 0$, by Lemma 2.2,

$$
\delta_{B}(g) \leq \sum_{r, s \in T} \delta_{Q_{r}, Q_{s}}(g)=\sum_{\substack{r, s \in T \\ r \neq s}} \delta_{Q_{r}, Q_{s}}(g)+\sum_{r \in T} \delta_{Q_{r}}(g) \leq 2 \times 4+1 \times 3=11 .
$$

Case 2. $(m-1) b=2 m(m+1) a^{2}$ and $(m-1) b \neq-2 m(m+1) a^{2}$. Then

$$
g \notin\left(Q_{2 m}-Q_{m+1}\right) \cap\left(Q_{m(m+1)}-Q_{2 m}\right) .
$$

Moreover, if $g \in Q_{m+1}-Q_{2 m}$, then there exists $(u, v) \in \mathbb{Z}_{p}^{2}$ such that

$$
a=u-v, \quad b=(m+1) u^{2}-2 m v^{2} .
$$

Thus

$$
b=(-m+1) v^{2}+2(m+1) a v+(m+1) a^{2} .
$$

We have $m-1 \not \equiv 0 \bmod p$ and $(m-1) b=2 m(m+1) a^{2}$, thus

$$
((-m+1) v+(m+1) a)^{2}=2 m(m+1) a^{2}+(-m+1) b=0 .
$$

Thus, there is a unique $v$ satisfying (2.2), hence $\delta_{Q_{m+1}, Q_{2 m}}(g)=1$. Similarly, we can show that if $g \in Q_{2 m}-Q_{m(m+1)}$, then $\delta_{Q_{2 m}, Q_{m(m+1)}}(g)=1$. Hence, for $g \neq 0$, by Lemma 2.2,

$$
\delta_{B}(g) \leq \sum_{r, s \in T} \delta_{Q_{r}, Q_{s}}(g)=\sum_{\substack{r, s \in T \\ r \neq s}} \delta_{Q_{r}, Q_{s}}(g)+\sum_{r \in T} \delta_{Q_{r}}(g) \leq 2 \times 3+1 \times 5=11 .
$$

Case 3. $(m-1) b=-2 m(m+1) a^{2}$ and $(m-1) b \neq 2 m(m+1) a^{2}$. Then

$$
g \notin\left(Q_{m+1}-Q_{2 m}\right) \cap\left(Q_{2 m}-Q_{m(m+1)}\right) .
$$

Moreover, if $g \in Q_{2 m}-Q_{m+1}$, then $\delta_{Q_{2 m}, Q_{m+1}}(g)=1$; if $g \in Q_{m(m+1)}-Q_{2 m}$, then $\delta_{Q_{m(m+1)}, Q_{2 m}}(g)=1$. Hence, for $g \neq 0$, by Lemma 2.2,

$$
\delta_{B}(g) \leq \sum_{r, s \in T} \delta_{Q_{r}, Q_{s}}(g)=\sum_{\substack{r, s \in T \\ r \neq s}} \delta_{Q_{r}, Q_{s}}(g)+\sum_{r \in T} \delta_{Q_{r}}(g) \leq 2 \times 3+1 \times 5=11 .
$$

This completes the proof of Lemma 2.3. 
REMARK 2.4. Since the number of quadratic nonresidues $\bmod p$ is $(p-1) / 2 \geq 5$ for $p \geq 11$, there exists a quadratic nonresidue $m$ such that $m+1 \not \equiv 0 \bmod p$, $3 m+1 \not \equiv 0 \bmod p$ and $m+3 \not \equiv 0 \bmod p$.

LEMMA 2.5. Let $p(\geq 11)$ be prime and $m$ be a quadratic nonresidue of $p$ with $m+1 \not \equiv 0 \bmod p, \quad 3 m+1 \not \equiv 0 \bmod p$ and $m+3 \not \equiv 0 \bmod p . \quad$ Put $B=Q_{m+1} \cup$ $Q_{m(m+1)} \cup Q_{2 m}$ and $B^{\prime}=\varphi(B)$. Then $\sigma_{B^{\prime}}(n) \leq 16$ for all $n$ and $\delta_{B^{\prime}}(n) \leq 11$ for all $n \neq 0$. Moreover, for every integer $0 \leq n<2 p^{2}$, at least one of the six numbers $n-p, n, n+p, n+2 p^{2}-p, n+2 p^{2}, n+2 p^{2}+p$ is in $B^{\prime}+B^{\prime}$.

Proof. Let $g, g^{\prime}, h, h^{\prime} \in B$. It is easy to verify that $\varphi(g)+\varphi\left(g^{\prime}\right)=\varphi(h)+\varphi\left(h^{\prime}\right)$ is possible only if $g+g^{\prime}=h+h^{\prime}$ and that $\varphi(g)-\varphi\left(g^{\prime}\right)=\varphi(h)-\varphi\left(h^{\prime}\right)$ is possible only if $g-g^{\prime}=h-h^{\prime}$. That is, $\varphi$ cannot increase the values of $\sigma$ and $\delta$. By Lemma 2.3, we have $\sigma_{B^{\prime}}(n) \leq 16$ for all $n$ and $\delta_{B^{\prime}}(n) \leq 11$ for all $n \neq 0$.

Now take an arbitrary $n \in\left[0,2 p^{2}\right)$ and write it in the form

$$
n=a+2 p b, \quad 0 \leq a \leq 2 p-1,0 \leq b \leq p-1 .
$$

We can find $(x, y) \in B$ and $\left(x^{\prime}, y^{\prime}\right) \in B$ such that

$$
a \equiv x+x^{\prime} \bmod p, \quad b \equiv y+y^{\prime} \bmod p .
$$

We have

$$
\begin{gathered}
-(2 p-1) \leq x+x^{\prime}-a \leq 2(p-1), \\
-(p-1) \leq y+y^{\prime}-b \leq 2(p-1),
\end{gathered}
$$

thus $x+x^{\prime}-a=-p, 0, p$ and $y+y^{\prime}-b=0, p$.

Case 1. $x+x^{\prime}-a=-p$ and $y+y^{\prime}-b=0$. Then

$$
n-p=a+2 p b-p=x+2 p y+x^{\prime}+2 p y^{\prime} \in B^{\prime}+B^{\prime} .
$$

Case 2. $x+x^{\prime}-a=0$ and $y+y^{\prime}-b=0$. Then

$$
n=a+2 p b=x+2 p y+x^{\prime}+2 p y^{\prime} \in B^{\prime}+B^{\prime} .
$$

Case 3. $x+x^{\prime}-a=p$ and $y+y^{\prime}-b=0$. Then

$$
n+p=a+2 p b+p=x+2 p y+x^{\prime}+2 p y^{\prime} \in B^{\prime}+B^{\prime} .
$$

Case 4. $x+x^{\prime}-a=-p$ and $y+y^{\prime}-b=p$. Then

$$
n+2 p^{2}-p=a+2 p b+2 p^{2}-p=x+2 p y+x^{\prime}+2 p y^{\prime} \in B^{\prime}+B^{\prime} .
$$

Case 5. $x+x^{\prime}-a=0$ and $y+y^{\prime}-b=p$. Then

$$
n+2 p^{2}=a+2 p b+2 p^{2}=x+2 p y+x^{\prime}+2 p y^{\prime} \in B^{\prime}+B^{\prime} .
$$

Case 6. $x+x^{\prime}-a=p$ and $y+y^{\prime}-b=p$. Then

$$
n+2 p^{2}+p=a+2 p b+2 p^{2}+p=x+2 p y+x^{\prime}+2 p y^{\prime} \in B^{\prime}+B^{\prime} .
$$

This completes the proof of Lemma 2.5. 
LEMMA 2.6. Let $p(\geq 11)$ be prime and $m$ be a quadratic nonresidue of $p$ with $m+1 \not \equiv 0 \bmod p, \quad 3 m+1 \not \equiv 0 \bmod p$ and $m+3 \not \equiv 0 \bmod p . \quad$ Put $B=Q_{m+1} \cup$ $Q_{m(m+1)} \cup Q_{2 m}, B^{\prime}=\varphi(B)$ and $V=B^{\prime}+\left\{0,2 p^{2}-p, 2 p^{2}, 2 p^{2}+p\right\}$. Then $V \subset$ $\left[0,4 p^{2}\right)$ is a set with $|V| \leq 12 p$ and satisfies $\left[4 p^{2}, 6 p^{2}\right) \subseteq V+V, \sigma_{V}(n) \leq 256$ for all $n$ and $\delta_{V}(n) \leq 176$ for all $n$ with at most 11 exceptions.

Proof. Note that $B^{\prime} \subset\left[0,2 p^{2}-p\right)$, thus $V \subset\left[0,4 p^{2}\right)$. In addition $|V| \leq 4\left|B^{\prime}\right|=$ $4|B| \leq 12 p$.

Since

$$
\begin{gathered}
V+V=B^{\prime}+B^{\prime}+\left\{0,2 p^{2}-p, 2 p^{2}, 2 p^{2}+p, 4 p^{2}-2 p,\right. \\
\left.4 p^{2}-p, 4 p^{2}, 4 p^{2}+p, 4 p^{2}+2 p\right\},
\end{gathered}
$$

by Lemma 2.5 , we have $\left[4 p^{2}, 6 p^{2}\right) \subseteq V+V$, and $V$ is the union of four translated copies of $B^{\prime}$, hence

$$
\max \sigma_{V}(n) \leq 16 \max \sigma_{B^{\prime}}(n) \leq 16 \times 16=256 .
$$

Since

$$
V-V=B^{\prime}-B^{\prime}+\left\{0, \pm\left(2 p^{2}-p\right), \pm 2 p^{2}, \pm\left(2 p^{2}+p\right), \pm p, \pm 2 p\right\},
$$

by Lemma 2.5 ,

$$
\delta_{V}(n) \leq 16 \times \max \delta_{B^{\prime}}(n) \leq 16 \times 11=176,
$$

unless $n=0, \pm\left(2 p^{2}-p\right), \pm 2 p^{2}, \pm\left(2 p^{2}+p\right), \pm p, \pm 2 p$.

This completes the proof of Lemma 2.6.

LEMMA 2.7. Let $X$ be a finite set of integers and $p(\geq 11)$ be a prime. There is a set $Y$ such that

$$
Y \subset\left(\frac{7 p^{2}}{8}, 5 p^{2}\right), \quad|Y| \leq 12 p, \quad\left[6 p^{2}, \frac{31}{4} p^{2}\right) \subset Y+Y,
$$

and

$$
D(X \cup Y)<D(X)+\frac{96}{p}|X|^{3}+864|X|^{2}+6672 p|X|+73728 p^{2} .
$$

Proof. Let $V$ be the set of Lemma 2.6 and put $Y=V+t$ where $t$ is an integer in $\left(7 p^{2} / 8, p^{2}\right]$. Equation (2.3) holds for any choice of $t$; we show that (2.4) holds for a suitable choice of $t$.

Let $Z=X \cup Y . D(Z)$ is the number of quadruples $\left(z_{1}, z_{2}, z_{3}, z_{4}\right)$ of elements of $Z$ satisfying

$$
z_{1}+z_{2}=z_{3}+z_{4}
$$

We split Equation (2.5) into the following five classes.

(a) All four unknowns are from $X$. This gives the term $D(X)$.

(b) One comes from $Y$, three from $X$. Equation (2.5) can be written as

$$
t=x_{1}+x_{2}-x_{3}-v, \quad v \in V .
$$


Let $S_{t}$ be the number of solutions,

$$
\sum_{7 p^{2} / 8<t \leq p^{2}} S_{t} \leq 12 p|X|^{3} .
$$

Hence

$$
\left(p^{2} / 8\right) \cdot \min S_{t}<\min S_{t} \cdot \sum_{7 p^{2} / 8<t \leq p^{2}} 1 \leq 12 p|X|^{3},
$$

thus

$$
\min S_{t} \leq \frac{96|X|^{3}}{p}
$$

(c) Two come from $Y$, two come from $X$.

Case 1. The unknowns $y_{1}$ and $y_{2}$ are on the same side. Equation (2.5) can be written as

$$
y_{1}+y_{2}=x_{1}+x_{2}, \quad y_{i} \in Y, x_{i} \in X .
$$

By Lemma 2.6, for every pair $x_{1}, x_{2}$, there are at most 256 solutions which give a total of $256|X|^{2}$. According to the position of the $y \mathrm{~s}$ in (2.5), the contribution of this term is at most $2 \times 256|X|^{2}=512|X|^{2}$.

Case 2. The unknowns $y_{1}$ and $y_{2}$ are on different sides, that is,

$$
y_{1}-y_{2}=x_{1}-x_{2}, \quad y_{i} \in Y, x_{i} \in X .
$$

By Lemma 2.6, if $x_{1}-x_{2}$ is none of the 11 exceptional numbers, then the contribution of this term is at most $2 \times 176|X|^{2}=352|X|^{2}$; if $x_{1}-x_{2}$ is one of the 11 exceptional numbers, then after fixing the value of $x_{1}-x_{2}$, the numbers $x_{1}$ and $y_{1}$ determine $x_{2}$ and $y_{2}$ uniquely, thus the contribution of this term is at most $4 \times 11 \times|X| \times|Y| \leq$ $528 p|X|$.

(d) Three come from $Y$, one comes from $X$. Equation (2.5) can be written as

$$
y_{1}+y_{2}=y_{3}+x, \quad y_{i} \in Y, x \in X .
$$

In this case, the contribution of this term is at most $2 \times 256 \times|X| \times 12 p=6144 p|X|$.

(e) Four unknowns are from $Y$. The contribution of this term is at most $2 \times 256 \times$ $(12 p)^{2}=73728 p^{2}$.

Hence

$$
D(X \cup Y)<D(X)+\frac{96}{p}|X|^{3}+864|X|^{2}+6672 p|X|+73728 p^{2} .
$$

This completes the proof of Lemma 2.7 .

Proof of TheOrem 1.1. By Lemma 2.1, for $x \geq 1342$, there is a prime $p$ for which $x<p<1.0147 x$. Thus we can take a sequence $p_{1}, p_{2}, \ldots$ of primes such that $p_{1}=1361$ and $1.12 p_{i}<p_{i+1} \leq 1.0147 \times 1.12 p_{i}<\sqrt{\frac{31}{24}} p_{i}$ for all $i$, that is, $1.12<$ $p_{i+1} / p_{i}<\sqrt{\frac{31}{24}}$ for all $i$. This ensures that the intervals $\left[6 p_{i}^{2}, \frac{31}{4} p_{i}^{2}\right.$ ) overlap and 
together cover $\left[6 p_{1}^{2},+\infty\right)$. Applying Lemma 2.7 to $p=p_{i}$, we get the set $Y_{i}$. Let $X_{0}=\left[0,6 p_{1}^{2}\right]$ and $X_{i}=X_{i-1} \cup Y_{i}$. Then $A=\bigcup_{i=0}^{\infty} X_{i}$ will be a basis of $\mathbb{N}$.

For $N \geq 7.628517798 \times 10^{27}>\frac{1}{2}\left(6 p_{1}^{2}+1\right)^{4}$, there exists an $i>1$ such that $p_{i}^{2}<2 N<p_{i+1}^{2}$, so

$$
\begin{aligned}
\left|X_{i-1}\right| & \leq\left|X_{0}\right|+12\left(p_{1}+p_{2}+\cdots+p_{i-1}\right) \\
& =\left|X_{0}\right|+12 p_{i}\left(\frac{25}{28}+\cdots+\left(\frac{25}{28}\right)^{i-1}\right) \\
& <101 p_{i} .
\end{aligned}
$$

By Lemma 2.7,

$$
\begin{aligned}
D\left(X_{i}\right) & =D\left(X_{i-1} \cup Y_{i}\right) \\
& <D\left(X_{i-1}\right)+\frac{96}{p_{i}}\left|X_{i-1}\right|^{3}+864\left|X_{i-1}\right|^{2}+6672 p_{i}\left|X_{i-1}\right|+73728 p_{i}^{2} \\
& <D\left(X_{i-1}\right)+108470160 p_{i}{ }^{2} .
\end{aligned}
$$

By induction,

$$
\begin{aligned}
D\left(X_{i}\right) & <D\left(X_{0}\right)+108470160\left(p_{i}^{2}+\cdots+p_{1}^{2}\right) \\
& =D\left(X_{0}\right)+108470160 p_{i}^{2}\left(1+\left(\frac{25}{28}\right)^{2}+\cdots+\left(\frac{25}{28}\right)^{2 i-2}\right) \\
& <\left(6 p_{1}^{2}+1\right)^{4}+534846576 p_{i}^{2} \\
& <534846577 p_{i}^{2} .
\end{aligned}
$$

Therefore,

$$
\sum_{n \leq N} \sigma_{A}^{2}(n) \leq D\left(X_{i}\right)<534846577 p_{i}^{2} \leq 1069693154 N
$$

This concludes the proof.

\section{References}

[1] Y. G. Chen, 'The analogue of Erdős-Turán conjecture in $\mathbb{Z}_{m}$ ', J. Number Theory 128 (2008), 2573-2581.

[2] P. Erdős, 'On a problem of Sidon in additive number theory', Acta Sci. Math. (Szeged) 15 (1954), 255-259.

[3] P. Erdős and P. Turán, 'On a problem of Sidon in additive number theory, and on some related problems', J. Lond. Math. Soc. 16 (1941), 212-215.

[4] L. Panaitopol, 'Inequalities concerning the function $\pi(x)$ : applications', Acta Arith. 94(4) (2000), 373-381.

[5] I. Z. Ruzsa, 'A just basis', Monatsh. Math. 109 (1990), 145-151.

[6] M. Tang, 'A note on a result of Ruzsa', Bull. Aust. Math. Soc. 77 (2008), 91-98.

MIN TANG, Department of Mathematics, Anhui Normal University,

Wuhu 241000, PR China

e-mail: tmzzz2000@163.com 